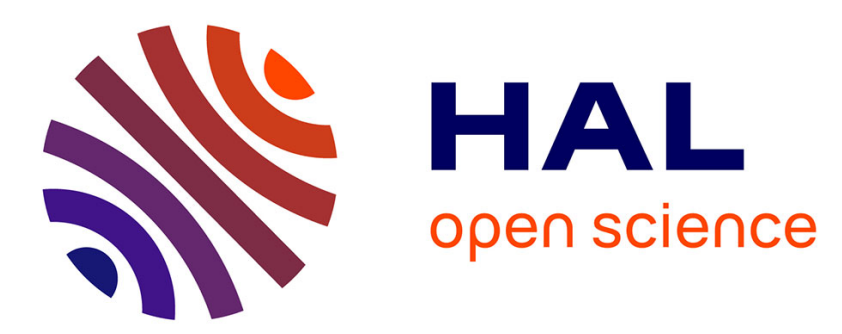

\title{
Differential coding in networked controlled linear systems
}

Carlos Canudas de Wit, Francisco Rubio, Jose Fornes, Fabio Gomez Estern

\section{To cite this version:}

Carlos Canudas de Wit, Francisco Rubio, Jose Fornes, Fabio Gomez Estern. Differential coding in networked controlled linear systems. American Control Conference, IEEE ACC'06, Jun 2006, -, France. pp.06. hal-00130509

\section{HAL Id: hal-00130509 https://hal.science/hal-00130509}

Submitted on 12 Feb 2007

HAL is a multi-disciplinary open access archive for the deposit and dissemination of scientific research documents, whether they are published or not. The documents may come from teaching and research institutions in France or abroad, or from public or private research centers.
L'archive ouverte pluridisciplinaire HAL, est destinée au dépôt et à la diffusion de documents scientifiques de niveau recherche, publiés ou non, émanant des établissements d'enseignement et de recherche français ou étrangers, des laboratoires publics ou privés. 


\title{
Differential Coding in Networked Controlled Linear Systems
}

\author{
Carlos Canudas-de-Wit, Francisco R. Rubio, José Fornés, and Fabio Gómez-Estern
}

\begin{abstract}
This paper investigates the closed-loop properties of the differential coding scheme known as Delta-Modulation $(\Delta-M)$ when used in feedback loops within the context of linear systems controlled through some communication network. We propose a new modified scheme of the original form of the $\Delta$ $M$ algorithm which improves the closed-loop properties. The new coding structure explicitly uses information from the system model. Stability properties for both: the continuoustime and the discrete-time version of this new algorithm are assessed. The results shows that the stability domain and the resulting precision of the $\Delta-M$ is limited by the position of the unstable poles of the system.
\end{abstract}

Index Terms-Differential coding, delta modulation, stabilization of linear systems in Networked controlled systems.

\section{INTRODUCTION}

This paper studies different aspects of the use of differential coding in as a mean for transmitting sensing signals in feedback controlled linear systems interconnected through some transmission network. The problem is of interest in the area of Networked Controlled Systems (NCS), where we find several applications calling for data-compression algorithms aiming at reducing the amount of information that may be transmitted throughout the communication channel, and therefore permitting a better resource allocation and/or an improvement of the permissible closed-loop system bandwidth (data-rate).

In particular, we study the closed-loop properties resulting from the Delta modulation $(\Delta-M)$ algorithm, which is the simplest form of the differential coding, in which a twolevel (1-bit) quantizer is used in conjunction with a firstorder predictor. As such, the $\Delta-M$ algorithm can be seen as special case of the family of signal quantizers. Although quantization may not be the largest factor contributing to data compression, and hence to high data rates, optimization of the quantization levels is mandatory in large-scale systems, and may be of great interest while designing low cost transmitter/receiver components [3].

Quantized feedback design, is a current topic of interest in the NCS field, see for instance [2], [6], [4], [8], [3], [5].

The $\Delta-M$ algorithm is a two-level dynamic quantizer, which can be seen as the coarsest dynamic quantizer, although this does not necessarily mean that the $\Delta$ - $M$ encoder yields the minimum data-rate stabilizing control strategy. The

C. Canudas-de-Wit is with The Laboratoire d'Automatique de Grenoble, UMR CNRS 5528, ENSIEG-INPG, B.P. 46, 38 402, ST. Martin d'Hères, FRANCE. Email: carlos.canudas-de-wit@inpg.fr.

Francisco R. Rubio José Fornés, and Fabio Gómez-Estern are with the Department of Automatic Control and Systems Engineering at the University of Sevilla, Spain. Email: rubio@esi.us.es, fornes@trajano.us.es, fabio@esi.us.es

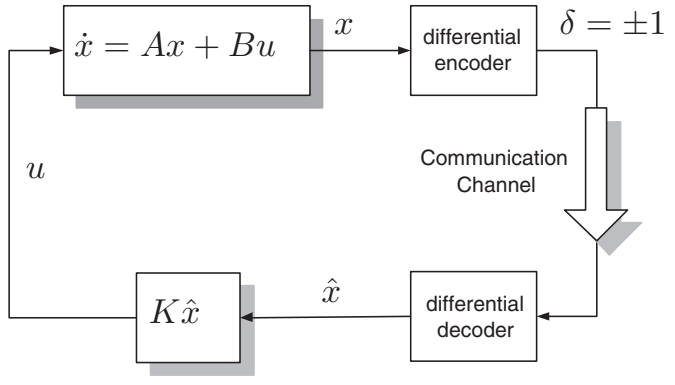

Fig. 1. Block diagram of the problem set up studied in this paper.

dynamic property of the Delta modulation, and its variants ${ }^{1}$ have been designed mainly from the signal transmission perspective, but no much as part of a feedback system, and as a consequence not much is known about the ability of this algorithm in the NCS context. This paper is devoted to this problem framed as shown in Figure 1.

First we present several results concerning the continuoustime formulation, which is relevant because it captures the limiting case of the discrete-time formulation, and provides a better understanding of the maximal stability properties that can be attained. Then, we study the discrete-time case, which permits a more clear assessment of the data rate constraints. It is shown that the system states converge to a ball enclosing the origin, and that the result is semi-global. The size of the attraction region, i.e. the system precision, depends on the coding gain linearly, and are also functions of the location of the unstable discrete-time open-loop poles. These results also show that the stability properties improve as the sampling time is reduced, or equivalently as the data rate is increased.

\section{A. Assumptions}

The hypothesis used in the results all along the paper, are the following:

- The transmitted information is binary $\delta_{k} \in\{-1,1\}$

- Only encoder-to-decoder information transmission is allowed (feedback between decoder to encoder is forbidden),

- Reliable noiseless channel transmission is considered (no data lost, or information distortion is assumed),

- Transmission delays are neglected,

- Channel rate capacity is $R$ [b.p.s.],

- Data is sent at a bounded rate (we select the sampling frequency in order to transmit only one $\delta_{k}$ at a time).

\footnotetext{
${ }^{1}$ Adaptive Delta modulation $(\mathrm{A} \Delta-M)$, and Sigma-Delta modulation $(\Sigma \Delta-M)$.
} 


\section{B. Definitions and notation}

Let $x \in \mathbb{R}^{n}, A \in \mathbb{R}^{n \times n}$, then : $\|x\|^{2}=\sum_{i=1}^{n} x_{i}^{2}$, $|x|=\sum_{i=1}^{n}\left|x_{i}\right|$, where $-|x| \leqslant-\|x\|, \operatorname{sgn}(x)=$ $\left[\operatorname{sgn}\left(x_{1}\right), \operatorname{sgn}\left(x_{2}\right), \cdots, \operatorname{sgn}\left(x_{n}\right)\right]^{T} .\|A\|$ is the induced Euclidian norm of $A$.

\section{Continuous-TIME FORMUlAtion}

We consider linear systems, with a linear feedback of the following form:

$$
\begin{aligned}
\dot{x}(t) & =A x(t)+B u(t) \\
u(t) & =-K \hat{x}(t)
\end{aligned}
$$

where $x(t) \in \mathbb{R}^{n}, A \in \mathbb{R}^{n \times n}, B \in \mathbb{R}^{n \times 1}$ are controllable matrices, $u(t)$ is the input of dimension one, $\delta(t) \in \mathbb{R}^{n}$ is the encoded signal, with only two-valued elements $\delta_{i}(t) \in$ $\{-1,1\} . \hat{x}(t) \in \mathbb{R}^{n}$ is the estimated value of $x$ as obtained from the process of differential coding described next. We consider here that the linear feedback gain $K$ is designed such that the matrix $A_{c}=A-B K$, is strictly stable. To simplify matters, we first consider a fully continuous-time formulation. The more realistic discrete-time case, including data-rate constraints, will be studied in latter sections.

This control setup is shown Figure 1, which describes a closed-loop system under one-way communication channel. The controller is assumed to be located at the plant side, whereas the sensor is remotely located. Information is transmitted through some data communication network. In order to focus the study on the impact of the differential coding, we only consider lossless transmission channels with neglected delay. Aspects related to the transmission delay have been studied elsewhere, see for instance [10], and [9].

\section{A. Differential coding}

Delta modulation $(\Delta-M)$ shown in Figure 2 is the simplest form of differential coding (see [7]), in which a two-level (1-bit) quantizer is used in conjunction with a first-order predictor. The continuous-time version of the $\Delta$ - $M$ coding algorithm is, with $\delta(t)=\operatorname{sgn}(x(t)-\hat{x}(t))$,

$$
\begin{aligned}
& \dot{\hat{x}}(t)=\Delta(t) \cdot \operatorname{sgn}(x(t)-\hat{x}(t))=\Delta(t) \cdot \delta(t) \\
& \hat{x}(t)=\int_{0}^{t} \Delta(\tau) \cdot \delta(\tau) d \tau
\end{aligned}
$$

The first equation (2a) describes the encoder dynamic equation, whereas the second one ( $2 \mathrm{~b})$ describes the decoder. $\Delta(t)$ is the step size, which in general is chosen to be constant, but time-varying gains can also be considered. A mandatory choice is that the gain $\Delta(t)$ must be the strictly the same in both sides of the coding process, in order to the decoder to work properly.

To simplify notation, in this section we drop the timearguments in all the variables when its explicit mention is not needed.

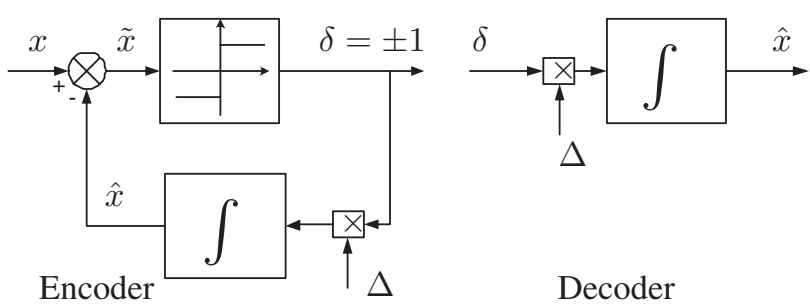

Fig. 2. Block diagram of standard continuous-time version of the $\Delta$ $M$ coder.

\section{B. Closed-loop stability properties of the standard $\Delta$ - $M$ coding algorithm}

Consider the problem of finding a suitable value for $\Delta$ so as to stabilize system (1), under the coding law (2). It is easy to see that the error equations read as:

$$
\begin{aligned}
\dot{x} & =A_{c} x+B K \tilde{x} \\
\dot{\tilde{x}} & =\underbrace{A_{c} x+B K \tilde{x}}_{\dot{x}}-\Delta \operatorname{sgn}(\tilde{x})
\end{aligned}
$$

with $\tilde{x}=x-\hat{x}$. Which means that $\Delta$ should be chosen large enough to locally dominate the rate of change of the system state, $\dot{x}$. Clearly, this can be done only under relative "high-values" for $\Delta$, as is demonstrated in the following result.

Proposition 1: STANDARD $\Delta-M$ CODING. Consider system (1) where $\hat{x} \in \mathbb{R}^{n}$ is the state estimate computed according to the $\Delta-M$ scheme shown in (2). Let $\tilde{x}=x-\hat{x}$, and assume that $\Delta \in \mathbb{R}^{n \times n}$ is constant, and has the following form:

$$
\Delta=\Delta_{0} \cdot I_{n \times n},
$$

Let $\zeta=\left(x^{T}, \tilde{x}^{T}\right)^{T}$, then for any possible value of initial conditions $\zeta(0)$, there exists a corresponding scalar constant value for $\Delta_{0}$ given by

$$
\Delta_{0} \geqslant c \cdot\|\zeta(0)\|
$$

such that $\zeta(t) \rightarrow 0$, as $t \rightarrow \infty$.

The constant $c>\max \left(c_{5}, c_{5} c_{6}\right)>0$, and the $c_{i}$ given by the following relations: $c_{1}=\|2 P B K\|$, $c_{2}=\left\|A_{c}\right\|, c_{3}=\|B K\|, c_{4}=\max \left\{c_{1}, c_{2}\right\}, c_{5}=c_{3}+\frac{c_{4}^{2}}{4 q}$, $c_{6}=\sqrt{\frac{\lambda_{\max } P+1}{\lambda_{\min } P+1}} \cdot \lambda_{\max } P, \lambda_{\min } P$, and $q=\lambda_{\min } Q$, are respectively, the maximum and minimum eigenvalues of $P=P^{T}>0$, and the minimum eigenvalue of $Q>0$, solutions of $P A_{c}+A_{c}^{T} P=-Q$.

Proof: Consider the quadratic Lyapunov function $V=$ $x^{T} P x+\tilde{x}^{T} \tilde{x}$, Evaluating $\dot{V}$ along solutions of system (3), and using $-|x| \leqslant-|| x||$, gives:

$$
\begin{aligned}
\dot{V} \leqslant & -q\|x\|^{2}+c_{1}\|x\|\|\tilde{x}\|+c_{2}\|x\|\|\tilde{x}\|+ \\
& +c_{3}\|\tilde{x}\|^{2}-\Delta_{0}|\tilde{x}| \\
\leqslant & -q\|x\|^{2}+c_{1}\|x\|\|\tilde{x}\|+c_{2}\|x\|\|\tilde{x}\|+ \\
& +c_{3}\|\tilde{x}\|^{2}-\Delta_{0}\|\tilde{x}\| \\
\leqslant & -q\|x\|^{2}+c_{4}\|x\|\|\tilde{x}\|+c_{3}\|\tilde{x}\|^{2}-\Delta_{0}\|\tilde{x}\|
\end{aligned}
$$




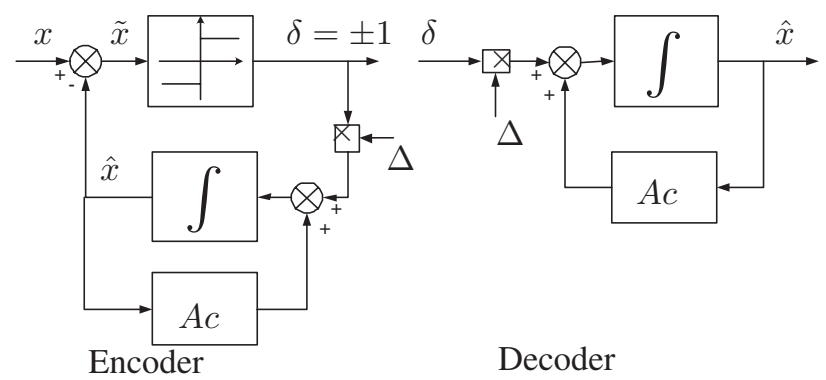

Fig. 3. Block diagram of standard continuous-time version of the $\Delta$ $M$ coder with the proposed modification.

$$
\leqslant-(\|x\|,\|\tilde{x}\|)\left(\begin{array}{cc}
q & -\frac{c_{4}}{2} \\
-\frac{c_{4}}{2} & \frac{\Delta_{0}}{\|\tilde{x}\|}-c_{3}
\end{array}\right)\left(\begin{array}{c}
\|x\| \\
\|\tilde{x}\|
\end{array}\right)
$$

From here we can see that $\dot{V}$ is negative as long as the matrix in the equality above is positive definite, i.e. for values of $\Delta_{0}$, such that $q\left(\frac{\Delta_{0}}{\|\tilde{x}\|}-c_{3}\right)>\frac{c_{4}^{2}}{4}$, or equivalently if $\Delta_{0}>c_{5}\|\zeta\| \geqslant c_{5}\|\tilde{x}\|$. Nevertheless, this condition by itself does not define the domain of attraction. For this we proceed further as follows.

Define $\lambda_{M}=\lambda_{\max } P+1$, and $\lambda_{m}=\lambda_{\min } P+1$. With this definition we have the following bounds on $V(\zeta)$,

$$
\begin{gathered}
\lambda_{M}\|\zeta\|^{2} \geqslant V(\zeta) \geqslant \lambda_{m}\|\zeta\|^{2} \\
-\lambda_{M}\|\zeta\|^{2} \leqslant-V(\zeta) \leqslant-\lambda_{m}\|\zeta\|^{2}
\end{gathered}
$$

using these bounds, and assuming that $\Delta_{0}>c_{5}\|\zeta\|$, then there exists a scalar function $\epsilon(\|\zeta\|)>0$, such that

$$
\dot{V} \leqslant-\epsilon(\|\zeta\|) \cdot\|\zeta\|^{2} \leqslant-\epsilon(\|\zeta\|) \frac{V}{\lambda_{M}}
$$

Integration on both sides of this equation along the timeinterval $[0, t]$, gives,

$$
V(t) \leqslant V(0) \exp (-\varphi(\epsilon(\|\zeta\|))
$$

where $\varphi(t)=\int_{0}^{t} \frac{\epsilon(\|\zeta(\tau)\|)}{\lambda_{M}} d \tau$. Note that $\varphi(t)>0$, as long as $\Delta_{0}>c_{5}\|\zeta\|$. Using again the bound on $V$ in the expression above, we obtain

$$
\begin{aligned}
\|\zeta(t)\|^{2} & \leqslant \frac{\lambda_{m}}{\lambda_{M}}\|\zeta(0)\|^{2} \exp (-\varphi(t)) \\
\|\zeta(t)\| & \leqslant \sqrt{\frac{\lambda_{m}}{\lambda_{M}}}\|\zeta(0)\| \exp (-\varphi(t)) \\
& =c_{6}\|\zeta(0)\| \exp (-\varphi(t) / 2),
\end{aligned}
$$

and using the relation given in Proposition 1, i.e. $\Delta_{0} \geqslant$ $c \cdot\|\zeta(0)\|>c_{5} c_{6}\|\zeta(0)\|$ in the above inequality, we get $\|\zeta(t)\|<\frac{\Delta_{0}}{c_{5}} \exp (-\varphi(t) / 2)$. Therefore, under the relation $\Delta_{0}>c_{5}\|\zeta(0)\|$, as proposed in Proposition 1, and using $\exp (-\varphi(t) / 2)<1 \forall t$, we conclude that $\mid \zeta(t) \|<\Delta_{0} / c_{5}$ as required for Eq. (4) to hold. As a final consequence, the time derivative of the Lyapunov function is negative definite and the convergence $\|\zeta(t)\| \rightarrow 0$ is guaranteed.

\section{A new structure for the $\Delta-M$ coding}

The result in Proposition 1 shows that relative large gains for $\Delta_{0}$ will be required to stabilize the system. Note that in this continuous-framework high gains will result in a important "chattering" effect, thus increasing the estimation error variance when the algorithm is discretized, or adding noise to the measured signal. Although the analysis is conservative, and probably lower values $\Delta_{0}$ may still be possible, it is interesting to study new structures of the $\Delta$ - $M$ coding such as to reduce the necessary gain for stabilization. The algorithm presented next is a modification of the original form of the $\Delta-M$ algorithm.

Consider now the following modified $\Delta-M$ algorithm together with system (1):

$$
\dot{\hat{x}}(t)=A_{c} \hat{x}+\Delta(t) \cdot \delta(t)
$$

with, $\delta(t)=\operatorname{sgn}(x(t)-\hat{x}(t))$, where a new term $A_{c} \hat{x}$ has been included. This equation describes both: the encoder and the decoder, as shown the block diagram of Figure 3.

It can be shown that the new error equation resulting from this new structure is described as two systems in cascade interconnection, i.e.

$$
\begin{aligned}
\dot{x} & =A_{c} x+B K \tilde{x} \\
\dot{\tilde{x}} & =A \tilde{x}-\Delta \operatorname{sgn}(\tilde{x})
\end{aligned}
$$

note that equation (6b) describes an autonomous system which solution is the input of the stable linear system (6a).

The stability properties of this algorithm are simplest than the one presented previously. They are also more tractable, and certainly less conservatives. They are given next, and also results in a system (6) which is semi-globally asymptotically stable.

Proposition 2: Modified $\Delta-M$ CODING. Consider system (1) together with the modified $\Delta-M$ coding scheme (5). As before, assume that $\Delta \in \mathbb{R}^{n \times n}$ is constant and has the following form:

$$
\Delta=\Delta_{0} \cdot I_{n \times n},
$$

and that $\Delta_{0}$ fulfills the following inequality,

$$
\Delta_{0}>a \cdot\|\tilde{x}(0)\|, \quad a=\frac{1}{2} \lambda_{\text {sup }}\left\{A+A^{T}\right\},
$$

then $\zeta(t)=\left(x^{T}(t), \tilde{x}^{T}(t)\right)^{T}$ is bounded and tends to zero as $t \rightarrow \infty$.

Proof: The proof is straightforward. Let $V=\tilde{x}^{T} \tilde{x} / 2$, and from equation $(6 b)$, we have that

$$
\begin{aligned}
\dot{V} & =\frac{1}{2} \tilde{x}^{T}(A \tilde{x}-\Delta \operatorname{sgn}(\tilde{x}))+\frac{1}{2}(A \tilde{x}-\Delta \operatorname{sgn}(\tilde{x}))^{T} \tilde{x} \\
& \leqslant-\Delta_{0}|\tilde{x}|+\frac{1}{2} \tilde{x}^{T}\left(A+A^{T}\right) \tilde{x} \\
& \leqslant-\Delta_{0}\|\tilde{x}\|+\frac{1}{2} \tilde{x}^{T}\left(A+A^{T}\right) \tilde{x} \\
& \leqslant-\Delta_{0}\|\tilde{x}\|+a\|\tilde{x}\|^{2}=-\|\tilde{x}\|\left(\Delta_{0}-a\|\tilde{x}\|\right)
\end{aligned}
$$

where we have used the relation $-|x| \leqslant-\|x\|$. From here we can see that the condition $\Delta_{0}>a \cdot\|\tilde{x}(0)\|$, 
with $a$ given as in the last Proposition, makes $V$ decrease, and hence ensures that $\|\tilde{x}(t)\|<\|\tilde{x}(0)\|$, and that $\tilde{x}(t)$ remains bounded and tends to zero in finite time. Finite-time convergence is typical in switching systems of the form (6b), and will not be demonstrated here. From this analysis we can also conclude that $\tilde{x}(t) \in \mathcal{L}_{2} \cap \mathcal{L}_{\infty}$.

To complete the proof note that equation (6b), describes an strictly stable linear system with input $\tilde{x}(t) \in \mathcal{L}_{2} \cap \mathcal{L}_{\infty}$, because $\tilde{x}(t)$ is bounded and tends to zero, we can conclude that $x(t)$ is also bounded and tends to zero, as the Proposition states.

Remark 1: Note that the proposed new coding form, in addition to simplify the stability conditions by making them only depend on the estimation initial error, the new structure introduces a side effect a low pass filtering action than will improve the filtering properties of the decoding dynamic equations.

Remark 2: Clearly, it is also interesting to study the case where only output signals $y \in \mathbb{R}^{m}$, are measurable. This case has also been analyzed and a further modified coding algorithm can be designed, see [1]

\section{DisCRETE-TIME Algorithm}

In this section we present the extension of the continuoustime differential coding to the more realistic case of discretetime framework. We first introduce a simple case of a onedimensional unstable system for which optimal gain $\Delta$, and attraction domain are easily found. We then extend this result to higher dimension systems.

\section{A. One-dimension system example}

Consider the following one-dimensional discrete timesystem, together with the control law, and the differential encoding modified law:

$$
\begin{aligned}
x_{k+1} & =a x_{k}+b u_{k} \\
u_{k} & =-K \hat{x}_{k} \\
\hat{x}_{k+1} & =a_{c} \hat{x}_{k}+\Delta \operatorname{sgn}\left(\tilde{x}_{k}\right)
\end{aligned}
$$

with, $K \in \mathbb{R}, a \geqslant 1, a_{c}=(a-b K) ;\left|a_{c}\right|<1$, and $\tilde{x}_{k}=$ $x_{k}-\hat{x}_{k}$.

The differential encoder (9) is designed on the same basis than the continuous-time version aiming at decoupling the coding error equation from the resulting closed-loop system. This gives the following error equations, with cascade structure:

$$
\begin{aligned}
& x_{k+1}=a_{c} x_{k}+b K \tilde{x}_{k} \\
& \tilde{x}_{k+1}=a \tilde{x}_{k}-\Delta \operatorname{sgn}\left(\tilde{x}_{k}\right)
\end{aligned}
$$

stability of the whole system can thus be tacked by only studying the stability of the coding error equation (11).

Let $V_{k}=\tilde{x}_{k}^{2}$, and $\nabla V_{k}=V_{k+1}-V_{k}$, then

$$
\begin{aligned}
\nabla V_{k} & =\tilde{x}_{k+1}^{2}-\tilde{x}_{k}^{2} \\
& =\left(a^{2}-1\right) \tilde{x}_{k}^{2}-2 a \Delta\left|\tilde{x}_{k}\right|+\Delta^{2}
\end{aligned}
$$

The right hand side of the equality defines a second order polynomial of the form $\alpha r^{2}+\beta r+\Delta^{2}=0$, with $r=\left|\tilde{x}_{k}\right|$ with roots $r_{1}, r_{2}$ given by:

$$
r_{1}=\frac{a-1}{a^{2}-1} \Delta, \quad r_{2}=\frac{a+1}{a^{2}-1} \Delta
$$

Note that the roots are always real and positive since $a>1$, and that these values defines three zones, where $\nabla V_{k}$ changes signs, i.e.

$$
\nabla V_{k}=\left\{\begin{array}{lll}
\geqslant 0 & \text { if } & \left|\tilde{x}_{k}\right| \leqslant r_{1} \\
<0 & \text { if } & r_{1}<\left|\tilde{x}_{k}\right|<r_{2} \\
\geqslant 0 & \text { if } & r_{2} \leqslant\left|\tilde{x}_{k}\right|
\end{array}\right.
$$

For some constant $\Delta$, this means that if the initial condition $\left|\tilde{x}_{0}\right|$, is taken within the region where $\nabla V_{k}$ is negative, then the function $V_{k}$ will decrease until $\left|\tilde{x}_{k}\right|$ enters in the region $\left|\tilde{x}_{k}\right|<r_{1}$, where $\nabla V_{k}$ changes sign. In other words, the error coding variable is locally attracted to a threshold delimited by the value of $r_{1}$, which depends linearly on the coding gain $\Delta$. This gains also delimits the stability border $r_{2}$, as shown the previous expressions.

Therefore, larger values for $\Delta$ will make the system more stable but less precise, and inversely reduction of the gain $\Delta$ will lead to small estimation error, but will at the same time reduce the domain where the system is keep stable.

We have proved the following result.

Proposition 3: DisCRETE ONE-DIMENSIONAL SYSTEM. Consider system (7)-(8), with constant $\Delta$, then if the initial conditions of the coding error are such that $\left|\tilde{x}_{0}\right|<r_{2}$ then the following hold:

- $\left|\tilde{x}_{k}\right|<r_{2}, \quad \forall k \geqslant 0$,

- $\exists k_{0}:\left|\tilde{x}_{k}\right| \leqslant r_{1}, \forall k \geqslant k_{0}$, and

- $\lim _{k \rightarrow \infty} d\left(x_{k}, \mathcal{B}_{\gamma}\right)=0$.

where $r_{1}$ and $r_{2}$ have been previously defined, and $d\left(x_{k}, \mathcal{B}_{\gamma}\right)$ is the minimum distance from $x_{k}$ to any point within the interval

$$
\mathcal{B}_{\gamma}:=\{x \in \mathbb{R}:|x|<\gamma\}, \quad \gamma=\frac{K b}{1-a_{c}} r_{1}
$$

Proof: The first two items result from the previous development. The last one derives from the following arguments. First note that $x_{k}=\frac{K b}{z-a_{c}} \tilde{x}_{k}$. Then, defining the discrete time transfer function $H(z)=\frac{K b z}{z-a_{c}}$, with impulse response $h(n)=K b a_{c}^{n}, n \geqslant 0$, yields the input-output relation $x_{k}=H(z) z^{-1} \tilde{x}_{k}$ which in time domain corresponds to the convolution

$$
x_{k}=\sum_{m=0}^{\infty} \tilde{x}_{m-1} h(k-m)=\sum_{m=0}^{\infty} \tilde{x}_{m} h(k-m-1)
$$

because $\tilde{x}_{m}=0 \forall m<0$ is assumed. Now using the fact that $x_{k}$ is absolutely bounded $\left(\left|\tilde{x}_{k}\right|<r_{2}, \forall k \geqslant 0\right)$ and also ultimately bounded by $r_{1}$ (second item of the Proposition), we can split the convolution as follows

$$
x_{k}=\sum_{m=0}^{k_{0}} \tilde{x}_{m} h(k-m-1)+\sum_{m=k_{0}+1}^{\infty} \tilde{x}_{m} h(k-m-1)
$$

hence

$$
\left|x_{k}\right| \leqslant r_{2} \sum_{m=0}^{k_{0}}|h(k-m-1)|+r_{1} \sum_{m=k_{0}+1}^{n-1}|h(k-m-1)|
$$


where the causality of $h(n)$ has been used. Substituting the impulse response into this expression yields

$$
\begin{aligned}
\left|x_{k}\right| & <K b r_{2}\left(\frac{a_{c}^{k}\left(1-a_{c}^{-k_{0}-1}\right)}{a_{c}-1}\right)+K b r_{1}\left(\frac{a_{c}^{k-k_{0}-1}-1}{a_{c}-1}\right) \\
& =\frac{K b r_{1}}{1-a_{c}}+v(k)
\end{aligned}
$$

where $v(k)$ stands for

$$
v(k)=\frac{K b\left(r_{2}\left(1-a_{c}^{-k_{0}-1}\right)+r_{1} a_{c}^{-k_{0}-1}\right)}{a_{c}-1} a_{c}^{k},
$$

and as $a_{c}<1$, we conclude that $\lim _{k \rightarrow \infty} v(k)=0$ and the state $x$ asymptotically approaches the interval $\left|x_{k}\right| \leqslant \gamma$ with

$$
\gamma=\frac{K b}{1-a_{c}} r_{1} .
$$

Remark 3: This result displays an inherent trade-off between stability and precision for discrete-time differential coding when the gain $\Delta$ is fixed. This suggests the search for other coding strategies with variant gains. Note also that, as the sampling time ${ }^{2}$ is chosen small, $a$ approaches 1 and the precision is increased. Indeed, $\lim _{a \rightarrow 1} r_{1}(a)=0$, thus by making $T_{s}$ infinitely small, the limit case of the continuoustime precision is approached.

Remark 4: The above analysis will be extended to the case where the open loop model is affected by bounded noise, see [1]

\section{B. n-dimensional systems}

The previous study can be generalized to system with higher dimension of the form

$$
\begin{aligned}
x_{k+1} & =A x_{k}+B u_{k} \\
u_{k} & =-K x_{k}
\end{aligned}
$$

where $x_{k} \in \mathbb{R}^{n}, A \in \mathbb{R}^{n \times n}, B \in \mathbb{R}^{n \times 1}$. $(A, B)$ are stabilizable pair.

For the sake of space, the noise considerations will be spared or simplicity, we consider systems whose matrix $A$ has distinct and real eigenvalues, that is there exist a transform matrix $T$ such that $\Lambda=T A T^{-1}=\operatorname{diag}\left\{\lambda_{i}\right\}$, with $\lambda_{i} \neq 0, \forall i$.

The differential encoding modified law is:

$$
\hat{x}_{k+1}=A_{c} \hat{x}_{k}+\Delta \operatorname{sgn}\left(T \tilde{x}_{k}\right)
$$

with, $A_{c}=(A-B K) ;\left|\lambda_{i}\left\{A_{c}\right\}\right|<1$, and $\tilde{x}_{k}=x_{k}-\hat{x}_{k}, \in$ $\mathbb{R}^{n}, \Delta \in \mathbb{R}^{n \times n}$. Note that the sign function depends on the transform coordinates $\tilde{z}_{k}=T \tilde{x}_{k}$, and

$\operatorname{sgn}\left(\tilde{z}_{k}\right)=\operatorname{sgn}\left(T \tilde{x}_{k}\right)=\left[\operatorname{sgn}\left(\tilde{z}_{1, k}\right), \operatorname{sgn}\left(\tilde{z}_{2, k}\right), \cdots \operatorname{sgn}\left(\tilde{z}_{n, k}\right)\right]^{T}$

The error equations in the $x_{k}$, and $\tilde{z}_{k}$ coordinates are:

$$
\begin{aligned}
x_{k+1} & =A_{c} x_{k}+B K T^{-1} \tilde{z}_{k} \\
\tilde{z}_{k+1} & =\Lambda \tilde{z}_{k}-T \Delta \operatorname{sgn}\left(\tilde{z}_{k}\right)
\end{aligned}
$$

\footnotetext{
${ }^{2}$ The pole of the discrete-time system is related to the pole, $\omega_{o l}$, of the open-loop continuous, one as $a=e^{\omega_{o l} T_{s}}$.
}

Proposition 4: DisCRETE N-DIMENSIONAL SYSTEM. Let $\lambda_{m}$, and $\lambda_{M}$, the smaller and the larger eigenvalues of $\Lambda$, respectively. Consider the differential encoding law (15), in feedback with system (13)-(14), with the gain $\Delta$ given as

$$
\begin{aligned}
\Delta & =\Delta_{0} \cdot T^{-1} \cdot \tilde{\Delta} \\
\tilde{\Delta} & =\operatorname{diag}\left\{\operatorname{sgn}\left(\lambda_{1}\right), \operatorname{sgn}\left(\lambda_{2}\right), \cdots, \operatorname{sgn}\left(\lambda_{n}\right)\right\}
\end{aligned}
$$

where $\Delta_{0}$ is a positive scalar constant. Then, if $\lambda_{m}^{2}>\left(\lambda_{M}^{2}-\right.$ 1 ), and the initial condition of the coding state are such that $\left\|\tilde{z}_{0}\right\|<r_{2}$ the following holds:

- $\left\|\tilde{z}_{k}\right\| \leqslant r_{2}, \quad \forall k \geqslant 0$

- $\exists k_{0}:\left|\tilde{z}_{k}\right| \leqslant r_{1}, \forall k \geqslant k_{0}$, and

- $\lim _{k \rightarrow \infty} d\left(x_{k}, \mathcal{B}_{\beta}\right)=0$.

where $0<r_{1}<r_{2}$ are given as:

$$
\begin{aligned}
& r_{1}=\frac{\Delta_{0}}{\lambda_{M}^{2}-1}\left[\lambda_{m}-\sqrt{\lambda_{m}^{2}-\lambda_{M}^{2}+1}\right] \\
& r_{2}=\frac{\Delta_{0}}{\lambda_{M}^{2}-1}\left[\lambda_{m}+\sqrt{\lambda_{m}^{2}-\lambda_{M}^{2}+1}\right]
\end{aligned}
$$

and $d\left(x_{k}, \mathcal{B}_{\beta}\right)$ is the minimum Euclidean distance from $x_{k}$ to any point within the ball

$$
\mathcal{B}_{\beta}:=\left\{x \in \mathbb{R}^{n}:\|x\|<\beta\right\},
$$

and $\beta$ is a constant that can be computed as in the proof of Proposition 3.

Proof: The proof follows along similar steps than the previous proof. Introduce $V_{k}=\tilde{z}_{k}^{T} \tilde{z}_{k}$, and $\nabla V_{k}=V_{k+1}-V_{k}$, then

$$
\begin{aligned}
\nabla V_{k}= & \tilde{z}_{k+1}^{T} \tilde{z}-\tilde{z}_{k}^{T} \tilde{z}_{k} \\
= & \tilde{z}_{k}^{T}\left(\Lambda^{2}-\mathcal{I}\right) \tilde{z}_{k}-2 \operatorname{sgn}\left(\tilde{z}_{k}\right) \tilde{\Delta} \Lambda \tilde{z}_{k}+ \\
& +\Delta_{0}^{2} \operatorname{sgn}\left(\tilde{z}_{k}\right)^{T} \tilde{\Delta}^{2} \operatorname{sgn}\left(\tilde{z}_{k}\right) \\
= & \tilde{z}_{k}^{T}\left(\Lambda^{2}-\mathcal{I}\right) \tilde{z}_{k}-2 \operatorname{sgn}\left(\tilde{z}_{k}\right) \tilde{\Delta} \Lambda \tilde{z}_{k}+\Delta_{0}^{2}
\end{aligned}
$$

Note that

$$
\begin{aligned}
-\operatorname{sgn}\left(\tilde{z}_{k}\right) \tilde{\Delta} \Lambda \tilde{z}_{k} & =-\sum_{i} \Delta_{0}\left|\tilde{z}_{i, k}\right| \cdot\left|\lambda_{i}\right| \\
& \leqslant-\Delta_{0} \lambda_{m} \sum_{i}\left|\tilde{z}_{i, k}\right|=-\Delta_{0} \lambda_{m}\left|\tilde{z}_{k}\right| \\
& \leqslant-\Delta_{0} \lambda_{m}|| \tilde{z}_{k}||
\end{aligned}
$$

Then,

$\nabla V_{k} \leqslant\left(\lambda_{M}^{2}-1\right)\left\|\tilde{z}_{k}\right\|^{2}-\Delta_{0} \lambda_{m}\left\|\tilde{z}_{k}\right\|+\Delta_{0}^{2} \equiv \varphi\left(\left\|\tilde{z}_{k}\right\|\right)$

$\varphi\left(\left\|\tilde{z}_{k}\right\|\right)=0$ defines a second order polynomial with roots, $r_{1}$, and $r_{2}$, as defined previously. A necessary condition for this polynomial to have real roots, or equivalently, for the minimum of $\varphi\left(\left\|\tilde{z}_{k}\right\|\right)$ to be negative is that $\lambda_{m}^{2}>\left(\lambda_{M}^{2}-1\right)$. However, this condition is only sufficient for making $\nabla V_{k}$ negative in the domain $r_{1} \leqslant\left\|\tilde{z}_{k}\right\| \leqslant r_{2}$. Other less conservative condition may be found.

We have, as before, three regions:

$$
\nabla V_{k}=\left\{\begin{array}{lll}
\geqslant 0 & \text { if } & \left\|\tilde{z}_{k}\right\| \leqslant r_{1} \\
<0 & \text { if } & r_{1}<\left\|\tilde{z}_{k}\right\|<r_{2} \\
\geqslant 0 & \text { if } & r_{2} \leqslant\left\|\tilde{z}_{k}\right\|
\end{array}\right.
$$



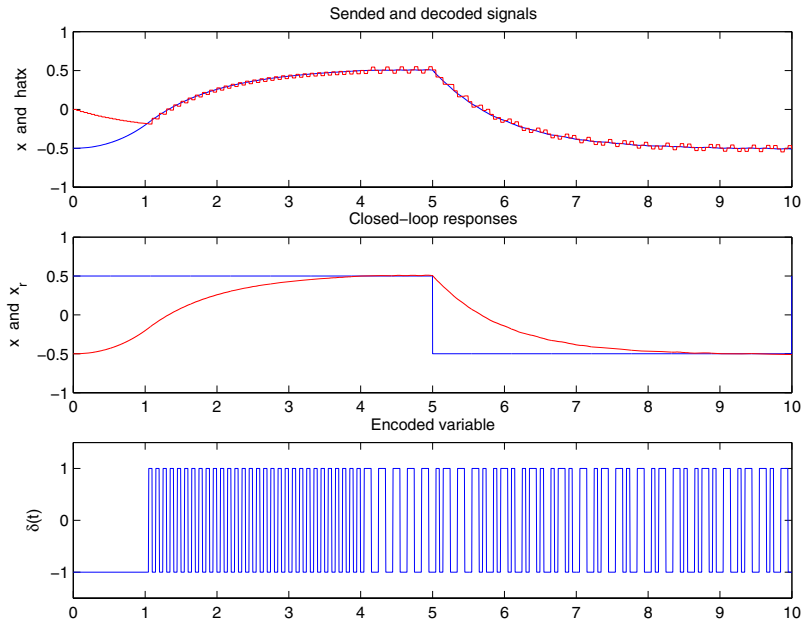

Fig. 4. Simulation results

and hence, the first two properties invoked in Proposition 4 follow exactly the same arguments than then ones used in previous sections. They are in consequence omitted here. The last statement follows from the relation $x_{k}=G(z) \tilde{z}_{k}$, and assuming that the $x$-subsystem (16) may also be diagonalized, the diagonalization leads to a set of $n$ perturbed systems of the form (10) in new coordinates, from which we conclude boundedness of the state $\left\|x_{k}\right\|$ based on the same arguments of Proposition 3 (omitted here for the sake of space).

\section{DATA-RATES}

It is assumed here that samples of $x(t)$, are digitalized with large enough resolution such that $x_{k}$ digitalization errors are neglected. As the transmission is done by only one bit, the data transmission rate (number of bits transmitted per unit of time) associated to this scheme, is $R_{\delta}=\frac{\text { one bit }}{T_{s}}=$ $f_{s}$, in $[$ Bits $/ \mathrm{sec}]$ where $f_{s}=1 / T_{s}$ is the sampling frequency, and the system precision is determined by the size of $\Delta$ quantizing the minimum step in the decoding process.

Let $R$, in $[$ Bits $/ \mathrm{sec}]$, be the network data-rate, then if the maximum network capabilities are used, i.e. $R_{\delta}=R=f_{s}$, the system precision and the domain of stability can be rewritten as a function of $R$. For instance, in the onedimensional case, the expression for $r_{1}$ and $r_{2}$ rewrites as:

$$
r_{1}=\frac{e^{\alpha / R}-1}{e^{2 \alpha / R}-1} \Delta, \quad r_{2}=\frac{e^{\alpha / R}+1}{e^{2 \alpha / R}-1} \Delta
$$

where $\alpha$ is the unstable pole of the continuous-time original system. As expected, increasing the transmission rate $R$, improves precision $\left(\lim _{R \rightarrow \infty} r_{1}(R)=0\right.$ ), and stability $\left(\lim _{R \rightarrow \infty} r_{2}(R)=\infty\right)$

\section{Simulations}

Simulations of the first order unstable system presented in Section III-A have been carried out with the following data: $a=e^{T_{s} \alpha}, \alpha=1, T_{s}=0.05, b=1-a, a_{c}=0.95$, and $\Delta=0.2$. The scheme has been adapted in such way that different set points can be tracked.
Figure 4 shows the obtained results. The upper figure shows the decoded signal $\hat{x}_{k}$, and the real signal $x(t)$. The initial condition for the estimated signal are $\hat{x}_{0}=0$, whereas the system is initialized at $x_{0}=-0.5$. As show this figure, the decoded is able to track reasonably well the source signal. Closed-loop responses are shown in the middle figure. We can observe that the so call granular noise (small oscillations around the source signal of amplitude proportional to the step size $\Delta$ ) do not have a major impact in the quality of the closed-loop responses. An explanation for that is the high frequency contains of the granular noise an its small amplitude. Finally the lower Figure shown the evolution in time of the one-bit modulated signal $\delta$.

\section{COnClusions}

In this paper we have investigated the stability properties of the Delta-modulation coding rule, when used as a transmission mean in networked controlled linear systems. It was first shown that the standard form of the $\Delta-M$ algorithm can be modified, including information about the system and the controller, to enhance the attraction domain to which the closed-loop system is shown to be stable. These results were extended to the case of output regulation, and finally we shown that in the discrete-time case, a trade-off between system precision and size of the stability domain can be assessed.

\section{ACKNOWLEDGEMENTS.}

Thanks are due to Prof. Sanjoy Mitter for their comments and discussion on the issue of this paper. The authors would like to acknowledge MCYT for funding this work under grants DPI2004-06419, HF2003-0237 and SAB20030085. Thanks are also due to the programm PICASSO No. 07261YJ(EGIDE), of the French Minister of foreign affaires.

\section{REFERENCES}

[1] Canudas-de-Wit C., F.R. Rubio, J. Fornes, and F. Gomez-Estern. Differential coding in networked controlled linear systems. Internal repport, see http://www.lag.ensieg.inpg.fr/canudas/, 2005.

[2] Elia N. and S.-K. Mitter. Stabilization of linear systems with limited information. IEEE Transaction on Automatic Control, 46(9):13841400, September 2001.

[3] Ishii H. and T. Başar. Remote control of lti systems over networks with state quatization. System and Control Letters, (54):15-31, 2005.

[4] Lemmon M. and Q. Ling. Control system performance under dynamic quatization: the scalar case. In 43rd IEEE Conference on Decision and Control, pages 1884-1888, Atlantis, Paradice Island, Bahamas, 2004.

[5] Li K. and J. Baillieul. Robust quatization for diginal finite communication bandwidth (dfcb) control. IEEE Transaction on Automatic Control, 49(9):1573-1584, September 2004.

[6] Liberzon D. On stabilization of linear systems with limited information. IEEE Transaction on Automatic Control, 48(2):304-307, February 2003.

[7] Proakis J.-G. Digital Communications. McGraw-Hill, Inc. Series in electrical and computer enginering.

[8] Tan S., Xi Wei, and J.-S. Baras. Numerical study of joint quatization and control under block-coding. In 43rd IEEE Conference on Decision and Control, pages 4515-4520, Atlantis, Paradice Island, Bahamas, 2004.

[9] Wiltrant E., C. Canudas de Wit, and D. Georges. "output stabilization via two-channel transmission communication". IFAC workshop on Time-Delay system, September 2003.

[10] Wiltrant E., C. Canudas de Wit, D. Georges, and M. Alamir. Remote stabilization via time-varying communication network delays. In IEEE Control application Conference, September 2004. 